\title{
Características morfológicas y tiempo de nado en púberes seleccionados del club Regatas, Lima
}

\author{
Morphological Characteristics and Swim Time in Publishers \\ Selected from the Lima Regatas Club
}

\section{HELDER FAVIO ÑAHUI ROJAS ${ }^{1}$}

https://orcid.org/0000-0002-3501-6913

\begin{abstract}
RESUMEN: Objetivo, determinar la existencia de influencia significativa de las características morfológicas sobre el tiempo de nado de deportistas entre los 11 y 13 años de edad. Métodos: La muestra estuvo conformada por 20 nadadores púberes de la selección del Club de Regatas "Lima". Para la recolección de los datos morfológicos se utilizó el protocolo que obedece a los estándares establecidos por la Sociedad Internacional para avances en Cineantropometría (ISAK) y para la toma de los tiempos de nado se utilizó un cronómetro manual de marca Robic con 1/100 segundo, precisión a 10 horas. Resultados: Existen diferencias significativas entre las características morfológicas de hombres y mujeres. En hombres se encontró correlación significativa entre la edad cronológica con el tiempo de nado y la velocidad de nado. En ningún caso se encontró correlación entre alguna característica morfológica con el tiempo de nado en los púberes. Conclusiones: Las características morfológicas no influyen en el tiempo de nado en púberes (11-13 años) de la selección del Club de Regatas "Lima".
\end{abstract}

Palabras clave: características morfológicas; natación; tiempo de nado.

ABSTRACT: To determine the existence of significant influence of the morphological characteristics on the swimming time of athletes between 11 and 13 years of age. Methods: The sample consisted of 20 pubescent swimmers from the selection of the Club of Regatas "Lima". For the collection of morphological data, the protocol that obeys the standards established by the International Society for Advances in Kineanthropometry (ISAK) was used and a manual Robic brand chronometer with 1/100 second was used for taking the swimming times, precision to 10 hours. Results: There are significant differences between the morphological characteristics of men and women. In men, a significant correlation was found between chronological age with swimming time and swimming speed. In no case was a correlation found between any morphological characteristic and swimming time in puberty. Conclusions: The morphological characteristics do not influence the swimming time in puberty (11-13 years) of the selection of the Club of Regatas "Lima".

Keywords: Morphology; Swimming; Swimming Time. 


\section{INTRODUCCIÓN}

La natación deportiva puede significar, en la vida del joven, una etapa muy rica en experiencias: continua confrontación con el medio acuático, como también goce de estas vivencias, esfuerzos de entrenamiento, alegría por los éxitos, decepciones por los fracasos, nuevas amistades (Wilke \& Madsen, 1990). En ese sentido debemos resaltar la importancia de las condiciones que van a permitir que ese individuo logre desarrollarse óptimamente en este deporte.

La morfología es la parte de la biología que trata de la forma de los seres orgánicos y de las modificaciones o transformaciones que experimenta (DRAE). Partiendo de ese enunciado las características de la forma corporal, además del tamaño absoluto, que exige cada deporte y que permiten obtener un desempeño óptimo pueden describirse mediante las proporciones morfológicas, es decir la relación de las partes del cuerpo con el resto o entre ellas (Abernethy et al. 1997; Norton \& Olds, 1996). Estos valores morfológicos pueden determinarse mediante la antropometría (Mazza, 2003).

En natación, la finalidad es recorrer una distancia determinada en el menor tiempo posible manteniendo el cuerpo en posición horizontal, por lo tanto, una estatura mayor y unas extremidades superiores proporcionalmente largas ofrecen ventajas (Norton \& Olds, 2002).

Uno de los problemas más importantes y difíciles de resolver en el ámbito de las ciencias del deporte es la dificultad de establecer puntualmente en qué medida contribuye el físico en el rendimiento deportivo (Carter, 2003). Parte de este problema se puede resolver a través de la cinenatropometría al relacionar el físico con el desempeño del atleta. De esta manera se puede obtener parte del conocimiento necesario para predecir el rendimiento deportivo que puede alcanzar un nadador (Abernethy et al., 1997).

Debido a que la morfología es una de las propiedades que está condicionada genéticamente ya que es muy poco modificable con el entrenamiento (Matveev, 2001), sólo es posible modificar los niveles de grasa corporal o los niveles de masa muscular, debemos tenerla en cuenta cuando nos referimos a la intención de obtener mejores nadadores. Además, existe una gran evidencia que muestra diferencias corporales entre atletas de deportes diferentes, o dentro del mismo deporte, pero en diversos eventos (Carter, 1982; Carter, 1984; Carter, 1985; Carter, 1994; Rienzi et al., 1995; Rivera \& Avella, 1992).

Es así que entre los factores que influyen en el rendimiento deportivo se incluye la morfología como medio para la valoración de la evolución y desarrollo del deportista. García Bataller (1999), divide en 5 apartados a valorar: mediciones antropométricas, valoración de la condición física general y específica, valoración técnica y psicológica. Más adelante, Arellano y de la Fuente (2000), los clasifican en: características físicas (peso, talla y longitud de segmentos corporales), fisiológicas (resistencia, velocidad de reacción, fuerza y flexibilidad), psicológicas y niveles de destreza. Saavedra et. al. (2002), construye una batería de valoración llamada META-NAT (Batería multidimensional de Evaluación de Talentos en la Natación; Saavedra, Escalante y Rodriguez 2001) en la que destacan seis apartados: Valoración de antecedentes sociales y deportivos; valoración somática; valoración de la condición física específica; valoración de la técnica; valoración multidimensional. Por 
tanto, la morfología, es considerada un factor importante a tener en cuenta en el rendimiento final del deportista (Ross et al., 1988).

Ahora, tenemos que determinar en qué momento a partir de la pubertad estas características morfológicas se pueden tomar en cuenta para la consecución de un mejor tiempo en el nado y así pues la identificación de futuros talentos deportivos en la natación. Boulgakova (1990) realiza un conjunto de análisis morfológicos y funcionales de los nadadores diferenciando sus características según los diferentes estilos y distancias de nado. Estudios del proceso de maduración en nadadores han mostrado que estos tienen medias superiores al resto de la población, diferencias entre ambos sexos y con respecto a otras especialidades deportivas (Damsgaard et al., 2001; Benefice et al., 1990) muestran una maduración sexual y esquelética avanzada (Malina, 1994); y a partir de los 13 años en niñas y 12 años en niñas un número importante de estas variables pueden usarse como estrategia para la identificación de talentos (Blanksby et al., 1994); Siders et al. (1993) por el contrario indican que las mediciones en composición corporal y somatotipo pueden ser un predictor del desarrollo sólo en el género femenino, no obteniendo correlaciones significativas para el género masculino.

Existen datos sobre las características morfológicas de los deportistas en algunos países del mundo, sobre todo en países desarrollados, pero en países en desarrollo como el Perú, el cual se encuentra en una etapa de mucha intención y esfuerzo por querer avanzar en temas científicos - deportivos, existe una carencia de estudios publicados y de datos propios de la realidad deportiva nacional. Esto nos muestra un déficit para elaborar de manera más certera determinadas estrategias en función al logro de objetivos en los diferentes deportes nacionales. Es por ello que se presenta este estudio que tiene como objetivo el determinar si existe una influencia significativa de las características morfológicas sobre el tiempo de nado de deportistas entre los 11 y 13 años de edad. Este estudio se vuelve pertinente en estos momentos, pues brindará datos de un sector deportivo, en este caso de la natación, así pues, poder seguir desarrollando este deporte de manera cada vez más eficiente.

\section{METODOLOGÍA}

La muestra fue de 20 nadadores del club de Regatas "Lima", 09 de género femenino y 11 de género masculino. Las edades de los nadadores fluctúan entre 11 y 13 años, todos pertenecientes a la categoría infantil B del mencionado club. Se incluyó en este estudio a nadadores que tenían por lo menos un año de entrenamiento regular con el equipo. El estudio se realizó previo consentimiento de la Dirección Técnica de Natación del club, y se obtuvo la autorización por parte de los padres y de los propios nadadores.

Tabla 1. Características generales.

\begin{tabular}{|r|c|c|}
\hline & Hombres & Mujeres \\
\hline Tamaño de la Muestra & 11 & 09 \\
\hline Edad (años) & $12.5 \pm 0.7$ & $12.8 \pm 0.8$ \\
\hline Talla (cm) & $155.5 \pm 4.6$ & $162.7 \pm 5.0$ \\
\hline Peso (kg) & $46.3 \pm 6.5$ & $50.8 \pm 8.5$ \\
\hline
\end{tabular}




\section{Características Morfológicas}

El protocolo usado para la recolección de datos sigue obedece a los estándares establecidos por la Sociedad Internacional para avances en Cineantropometría (ISAK) (Marfell-Jhones, 2001; Norton \& Olds, 1996), incluyendo para cada deportista, además las variables, masa corporal (peso muscular, peso graso, peso oseo); estatura; estatura sentada; envergadura; longitudes (acromio - dactilio, acromio - radial, radial - estilio, ilioespinal-suelo, ilioespinal - tibial, tibial - maleolar, de la mano y del pie) diámetro óseo (biacromial) y pliegues de tejido adiposo subcutáneo (subescapular, tricipital y de la pantorrilla media).

Los datos obtenidos, en total 16 , fueron recogidos por personal capacitado, quienes contaban con la certificación Nivel I, otorgadas por la ISAK, y por lo tanto tenían un error técnico de medición dentro de márgenes aceptables para este tipo de estudios $(5 \%$ para pliegues cutáneos y $2 \%$ para el resto de las medidas antropométricas).

Los instrumentos de evaluación utilizados incluyeron: balanza digital (Tanita), con precisión de $100 \mathrm{~g}$; tallímetro con precisión de $1 \mathrm{~mm}$; antropómetros, segmómetro y cinta métrica (Rosscraft), con precisión de $1 \mathrm{~mm}$; calibre de pliegues cutáneos (Rosscraft), con precisión de $0.5 \mathrm{~mm}$.

\section{Tiempo de nado}

Se realizó activación fisiológica previa al test, la cual consistió en estiramientos activos (de extremidades y tronco) fuera de la piscina durante 15 minutos. Luego $400 \mathrm{~m}$. de nado a estilo libre con pulsaciones entre 160 y 180 por minuto.

Se consideró para los diferentes momentos en la toma de tiempos los siguientes criterios:

Tiempo de salida (TS): Tiempo que transcurre desde la señal de salida hasta que la cabeza del nadador pasa por una referencia situada a $10 \mathrm{~m}$ de la pared de salida en piscinas de 50 metros y a $15 \mathrm{~m}$ de la pared de salidas en piscinas de $25 \mathrm{~m}$. (Haljand, 1992).

Tiempo de nado (Tn): Está determinado por la distancia de la prueba y la velocidad media sobre dicha distancia. (Hay, 1985). La velocidad media del nadador es igual al producto de dos factores: la longitud de ciclo (Lc) y la frecuencia de ciclo (Fc). (Thayer y Hay, 1984).

Frecuencia de ciclo media $\left(F_{c}\right)$ : Es el número de ciclos de brazos $(c)$ realizados por el nadador en la unidad de tiempo. Se expresa en $\mathrm{Hz}$, aunque los entrenadores nos manejamos en ciclos por minuto, siendo esa la información que transmitimos al nadador.

$\mathrm{N}^{\mathrm{o}}$ de ciclos contados

$\mathrm{Fc}=$

Tiempo invertido en el $\mathrm{n}^{\mathrm{o}}$ de ciclos contados 
Longitud de ciclo media (Lc): Es el espacio horizontal recorrido hacia delante por el nadador en cada ciclo completo de brazos. (Pai, Hay y Wilson, 1984).

Espacio recorrido (m)

$$
\mathrm{Lc}=
$$

\section{$\mathrm{N}^{\mathrm{o}}$ de ciclos contados}

Tiempo de Viraje (Tv): Desde una perspectiva cinemática, es el tiempo que transcurre desde que la cabeza del nadador pasa por una referencia situada a 7,5 $\mathrm{m}$ de la pared de viraje, hasta que tras realizarlo vuelve a pasar la cabeza por esa misma referencia. (Arellano, De Aymerich, Sánchez, \& Rivera, 1993). El tiempo obtenido se descompone en fase de aproximación y fase de separación.

Tiempo de llegada (Tll): Es el transcurrido desde que la cabeza pasa por una referencia situada a 5 o 7,5 metros de la pared de llegadas en función del tamaño del vaso, hasta que el nadador contacta con la pared (Haljand, 1992).

El procedimiento es el siguiente: El cronometrador se desplaza paralelamente al sentido de nado tomando tiempo en las referencias situadas con respecto a la salida a:

10m: Se obtiene el tiempo de salida y velocidad de salida.

20m: Al restar el T10 al T20, obtenemos un tiempo en 10 metros sin influencias de salida ni de virajes por lo que obtenemos la velocidad media de nado del primer largo.

25m: Nos da información sobre el primer parcial de la prueba y restando T25 - T20 nos da el tiempo de la fase de aproximación del viraje.

30m: T30 - T25 nos da el tiempo de la fase de separación del viraje y T30 - T20 nos da el tiempo del viraje completo y por consiguiente la velocidad de viraje.

45m: T45 - T30 obtenemos un tiempo de nado en 15 metros sin influencias de salidas ni de virajes que nos permite determinar la velocidad media real de nado del $2^{\circ}$ parcial de la prueba.

50m: Nos da el tiempo total de duración de la prueba. T50 - T45 nos da el tiempo de llegada en 5 metros y la velocidad de llegada.

Para tomar cada tiempo se utilizó un cronómetro manual de marca Robic. Con las siguientes características: $1 / 100$ de segunda precisión a 10 horas, ficheros de memoria según las pruebas, borrado selectivo de memorias, visualización de tiempos intermedios y fraccionados entre otras funciones.

\section{Análisis Estadísticos}

Se verifico la normalidad de la distribución de cada una de las variables a través de la prueba estadística Shapiro - Wilk. Se realizó estadística descriptiva para determinar medias y desviaciones estándar mínimos y máximos de las características morfológicas y los resultados en la prueba de velocidad. Se utilizó la t de Student para hallar diferencias en las medidas antropométricas entre hombres y mujeres. Se utilizó el coeficiente de correlación 
de Pearson (r), para identificar la contribución de las características morfológicas al tiempo total obtenido en la prueba de nado. En todas las pruebas se consideró una significancia menor o igual a 0.05. Para estos análisis estadísticos se utilizó el programa SPSS 15.

\section{Resultados.}

En la tabla 2 mostramos los resultados obtenidos en las mediciones antropométricas, los cuales muestran las diferencias entre las características morfológicas del sexo masculino y femenino.

Tabla 2. Diferencias de las características morfológicas entre el sexo femenino y el sexo masculino.

\begin{tabular}{|c|c|c|c|c|}
\hline \multirow[t]{2}{*}{ Características Morfológicas } & \multicolumn{2}{|c|}{ Sexo Masculino $(\mathrm{n}=11)$} & \multicolumn{2}{|c|}{ Sexo femenino $(n=09)$} \\
\hline & Media \pm DS & Intervalo & Media \pm DS & Intervalo \\
\hline Talla (cm) & $155.5 \pm 4.6$ & $149-164$ & $162.7 \pm 5.0$ & $156-171.5$ \\
\hline Peso (kg) & $46.3 \pm 6.5$ & $36.6-56.4$ & $50.8 \pm 8.5$ & $40.4-70.3$ \\
\hline Envergadura $(\mathrm{cm})$ & $159.5 \pm 5.1$ & $152-167$ & $164 \pm 5.5$ & $154-172$ \\
\hline Talla sentado $(\mathrm{cm})$ & $80.9 \pm 2.0$ & $77.2-84.7$ & $85.2 \pm 3.3$ & $81.5-93$ \\
\hline Peso Muscular (kg) & $19.5 \pm 3.3$ & $15-23.3$ & $21.8 \pm 3.4$ & $18.5-29$ \\
\hline Peso Grasa (kg) & $10.4 \pm 4.4$ & $6.6-18.8$ & $12.0 \pm 5.8$ & $7.2-26.7$ \\
\hline Peso Óseo (kg) & $10.2 \pm 1.1$ & $8.2-11.5$ & $10.5 \pm 1.6$ & $8.9-14.1$ \\
\hline $\begin{array}{l}\text { Longitud acromio }- \text { dactilio } \\
\qquad(\mathrm{cm})\end{array}$ & $69.7 \pm 2.2$ & $66.3-73.3$ & $72.9 \pm 2.9$ & $68-78$ \\
\hline Longitud acromio - radial $(\mathrm{cm})$ & $30.7 \pm 1.3$ & $29-32.5$ & $30.3 \pm 1.3$ & $29-33$ \\
\hline Longitud radial - estilio $(\mathrm{cm})$ & $32.5 \pm 8.5$ & $21-40$ & $23.3 \pm 0.7$ & $22.5-25$ \\
\hline $\begin{array}{l}\text { Longitud ilioespinal - suelo } \\
\qquad(\mathrm{cm})\end{array}$ & $88.7 \pm 3.9$ & $84.5-95.5$ & $93.1 \pm 3.2$ & $89.3-98$ \\
\hline $\begin{array}{l}\text { Longitud ilioespinal - tibial } \\
\qquad(\mathrm{cm})\end{array}$ & $47.5 \pm 3.1$ & $43.5-52$ & $47.6 \pm 4.4$ & $39-55$ \\
\hline Longitud tibial - maleolar (cm) & $34.2 \pm 1.6$ & $32.3-38$ & $33.5 \pm 4.8$ & $23.5-42$ \\
\hline Longitud de la mano $(\mathrm{cm})$ & $17.4 \pm 0.9$ & $16.1-18.9$ & $18.5 \pm 0.8$ & $17.1-19.8$ \\
\hline Longitud del pie (cm) & $23.6 \pm 1.0$ & $22.1-25$ & $24.1 \pm 0.8$ & $23-25.7$ \\
\hline Diámetro biacromial (cm) & $34 \pm 1.6$ & $30.4-35.7$ & $35.1 \pm 2.1$ & $32.6-38.8$ \\
\hline
\end{tabular}

En la tabla 3 los datos son medias ( \pm , DS), valores mínimos y máximos (intervalos), y correlación Pearson $(\mathrm{r}) *=(\mathrm{p}<0.05) * *=(\mathrm{p}<0.01)$ se muestra el análisis de coeficiente de correlación - Pearson (r) para hombres, el cual mostró que sólo se encontró correlación significativa $p<0.05(*)$ en la edad cronológica con el tiempo de nado y $p<0.01$ (**) en el tiempo de nado y la velocidad de nado. En ningún otro caso se encontró correlación. 
Tabla 3. Parámetros antropométricos, y su relación con el tiempo de nado de $50 \mathrm{~m}$ en estilo libre en modalidad crawl en nadadores púberes, género masculino.

\begin{tabular}{ccccc}
\hline Variable & Media $( \pm$ DS) & Mínimo & Máximo & $\begin{array}{c}\text { Correlación de Pearson } \\
\text { (r) con el Tiempo de Nado } \\
\text { en 50 m. }\end{array}$ \\
\hline Edad (años) & $12.5 \pm 0.7$ & 11.7 & 13.5 & $-0.630^{*}$ \\
Talla (cm.) & $155.5 \pm 4.6$ & 149 & 164 & $-0,486$ \\
Peso (Kg.) & $46.3 \pm 6.5$ & 36.6 & 56.4 & $-0,167$ \\
Velocidad de Nado & $1.48 \pm 0.16$ & 1.13 & 1.68 & $-0,951^{* *}$ \\
Envergadura (cm.) & $159.5 \pm 5.1$ & 152 & 167 & $-0,444$ \\
Talla sentado (cm.) & $80.9 \pm 2.0$ & 77.2 & 84.7 & $-0,281$ \\
Peso Muscular (Kg.) & $19.5 \pm 3.3$ & 15 & 23.3 & $-0,358$ \\
Peso Grasa (Kg.) & $10.4 \pm 4.4$ & 6.6 & 18.8 & 0,284 \\
Peso Óseo (Kg.) & $10.2 \pm 1.1$ & 8.2 & 11.5 & $-0,156$ \\
Longitud acromio - dactilio (cm.) & $69.7 \pm 2.2$ & 66.3 & 73.3 & $-0,243$ \\
Longitud acromio - radial (cm.) & $30.7 \pm 1.3$ & 29 & 32.5 & $-0,268$ \\
Longitud radial - estilio (cm.) & $32.5 \pm 8.5$ & 21 & 40 & 0,494 \\
Longitud ilioespinal - suelo (cm.) & $88.7 \pm 3.9$ & 84.5 & 95.5 & $-0,348$ \\
Longitud ilioespinal - tibial (cm.) & $47.5 \pm 3.1$ & 43.5 & 52 & $-0,362$ \\
Longitud tibial - maleolar (cm.) & $34.2 \pm 1.6$ & 32.3 & 38 & $-0,037$ \\
Longitud de la mano (cm.) & $17.4 \pm 0.9$ & 16.1 & 18.9 & $-0,380$ \\
Longitud del pie (cm.) & $23.6 \pm 1.0$ & 22.1 & 25 & $-0,337$ \\
Diámetro biacromial (cm.) & $34 \pm 1.6$ & 30.4 & 35.7 & $-0,569$ \\
\hline
\end{tabular}

En la tabla 4 los datos son medias ( \pm , DS), valores mínimos y máximos (intervalos), y correlación Pearson $(\mathrm{r}) *=(\mathrm{p}<0.05) * *=(\mathrm{p}<0.01)$, se muestra el análisis de coeficiente de correlación - Pearson (r) para mujeres, el cual mostró que sólo se encontró correlación significativa $\mathrm{p}<0.01(* *)$ en el tiempo de nado con la velocidad de nado. En ningún otro caso se encontró correlación. 
Tabla 4. Parámetros antropométricos y su relación con el rendimiento en el nado de $50 \mathrm{~m}$. en estilo libre en la modalidad crawl en nadadores púberes, género.

\begin{tabular}{|c|c|c|c|c|}
\hline Variable & Media ( \pm DS) & Mínimo & Máximo & $\begin{array}{c}\text { Correlación de } \\
\text { Pearson (r) con el } \\
\text { Tiempo de Nado en } \\
50 \mathrm{~m} .\end{array}$ \\
\hline Edad (años) & $12.8 \pm 0.8$ & 11.11 & 13.3 & -0.474 \\
\hline Estatura (cm.) & $162.7( \pm 5.0)$ & 149 & 164 & $-0,476$ \\
\hline Peso (Kg.) & $50.8 \quad( \pm 8.5)$ & 36.6 & 56.4 & 0,113 \\
\hline Velocidad de nado & $1.38( \pm 0.1)$ & 1.26 & 1.54 & $-0.899 * *$ \\
\hline Envergadura $(\mathrm{cm})$. & $164 \quad( \pm 5.5)$ & 152 & 167 & $-0,327$ \\
\hline Talla sentado $(\mathrm{cm})$. & $85.2( \pm 3.3)$ & 77.2 & 84.7 & $-0,370$ \\
\hline Peso Muscular (Kg.) & $21.8 \quad( \pm 3.4)$ & 15 & 23.3 & 0,067 \\
\hline Peso Grasa (Kg.) & $12.0 \quad( \pm 5.8)$ & 6.6 & 18.8 & 0,302 \\
\hline Peso Óseo (Kg.) & $10.5( \pm 1.6)$ & 8.2 & 11.5 & $-0,120$ \\
\hline Longitud acromio - dactilio (cm.) & $72.9 \quad( \pm 2.9)$ & 66.3 & 73.3 & $-0,401$ \\
\hline Longitud acromio - radial $(\mathrm{cm})$. & $30.3 \quad( \pm 1.3)$ & 29 & 32.5 & 0,403 \\
\hline Longitud radial - estilio (cm.) & $23.3 \quad( \pm 0.7)$ & 21 & 40 & $-0,485$ \\
\hline Longitud ilioespinal - suelo (cm.) & $93.1 \quad( \pm 3.2)$ & 84.5 & 95.5 & $-0,382$ \\
\hline Longitud ilioespinal - tibial (cm.) & $47.6( \pm 4.4)$ & 43.5 & 52 & $-0,593$ \\
\hline Longitud tibial - maleolar (cm.) & $33.5 \quad( \pm 4.8)$ & 32.3 & 38 & $-0,447$ \\
\hline Longitud de la mano (cm.) & $18.5( \pm 0.8)$ & 16.1 & 18.9 & $-0,492$ \\
\hline Longitud del pie (cm.) & $24.1 \quad( \pm 0.8)$ & 22.1 & 25 & 0,187 \\
\hline Diámetro biacromial (cm.) & $35.1 \quad( \pm 2.1)$ & 30.4 & 35.7 & $-0,026$ \\
\hline
\end{tabular}

\section{DISCUSIÓN}

Los hallazgos en el presente estudio sobre las diferencias morfológicas entre damas y varones púberes nos permitieron realizar el estudio por sexo. Mediante la verificación de posibles diferencias entre las características morfológicas de damas y varones se halló que, en la estatura, envergadura, estatura sentado, longitudes como el acromio - dactilio, radial - estilio, ilioespinal - suelo, y la longitud de la mano había diferencias significativas.

Los principales hallazgos de este estudio son que las características morfológicas en estas edades no juegan un rol protagónico en el tiempo de nado. Hemos encontrado, a través del análisis del coeficiente de correlación Pearson (r), que cuando analizamos los datos de los nadadores no muestra ninguna correlación entre las características morfológicas y el tiempo de nado o la velocidad de. Los resultados del presente estudio contrastan con los obtenidos por Osorio en el 2009 en el cual indica que la estatura y el diámetro de la mano tienen alta correlación con el tiempo de nado en varones entre 10 y 13 años. (Osorio, et al., 2009). Según los resultados de este estudio, los mejores tiempos de nado de nadadores púberes no se están en función a sus características morfológicas.

A través del presente estudio no negamos la influencia de las características morfológicas en el tiempo de nado en todas las categorías, sin embargo, determinamos, por los resultados 
encontrados, que dichas características no influyen en un mejor tiempo de nado en púberes (11 a 13 años) del club Regatas Lima.

\section{Conclusiones:}

Hay diferencias significativas entre las características morfológicas de los varones y las damas en nadadores púberes (11 a 13 años) del club de Regatas "Lima"

En individuos de ambos sexos, se encontró correlación entre el tiempo de nado y la velocidad de nado.

Las características morfológicas no tienen ninguna correlación con el tiempo de nado en nadadores púberes (11 a 13 años) de ambos sexos del club de Regatas "Lima".

Se encontró correlación significativa entre la edad cronológica y el tiempo de nado en varones, es decir a más edad mejoraba el tiempo de nado.

\section{REFERENCIAS}

Arellano, R., De Aymerich J., Sánchez J. A. y Rivera J. (1993). Análisis de la Actividad Competitiva en Natación. FINA Short Course $(25 \mathrm{~m})$ World Championships. Mallorca, España. Federación Española de Natación.

Arturo, O. Gutiérrez, Lidia De León F., Uriel Zúñiga G., (2009). Contribución de la morfología al rendimiento deportivo en nadadores niños y adultos. Efdeportes.com http://www.efdeportes.com/efd130/contribucion-de-la-morfologia-al-rendimientodeportivo-en-nadadores.htm

Barajas, Y. \& Santana F. (2010). Características morfológicas de los deportistas con altos logros de las selecciones de levantamiento de pesas, voleibol y karate-do del departamento de Córdoba, Colombia. Efdeportes.com, 15(148). http://www. efdeportes.com/efd148/caracteristicas-morfologicas-de-los-deportistas-con-altoslogros.htm

Boulgakova, N. (1990). Selection et preparation des jeunes nageurs. Editorial Vigot.

Cancela, J, \& Ramírez E. (2003). La formación de jóvenes nadadores. Evolución de la composición corporal y de los niveles de fuerza de desplazamiento en nadadores/as brasileños/as de edad comprendida entre los 13 y 23 años. Efdeportes.com, 9(65), http://www.efdeportes.com/efd65/nadador.htm

Carter, J. (1982). Physical structure of Olympic athletes. Part I: The Montreal Olympic Games anthropological project. Krager.

Carter, J. (1984). Physical structure of Olympic athletes. Part II: Kinantrophometry of Olympic athletes.

Carter, J. (1985). Morphological Factors limiting human performance. American Academy of. Physical Education Papers, (18), (edited by D.H. Clarcke and H.M. Eckert), pp.106 - 117. Champaign. Human Kinetics.

Carter, J., \& Ackland, T. (Eds) (1994). Kinanthropometry in Aquatic Sports: A study of world class athletes. Champaign. Human Kinetics. 
Carter, J. (1978). Predictions of outstanding athletic ability: The structural perspective In. F. Landry \& W. Orban (Eds.), Exercise Physiology. Vol. 4. Simposia Specialists.

Haljand, R. (1992). Competition Analysis in Swimming. European Research, 28.

Hay, J. (1985). Swimming. En J. Hay (Ed), The Biomechanics of sports Techniques (pp. 393-394). New Jersey - Hall International.

Malina, R., Martorell, R., \& Mendoza. F. (1986) Selected body measurements of children 6 - 11 years. Vital and Health Statistics, 11(123).

Matveev, L. (2001). Manifestación o expresión de la predisposición deportiva individual, orientación deportiva inicial y planteamiento de los objetivos a largo plazo en la preparación del deportista. En Teoría General del Entrenamiento. Editorial Paidotribo, pp. 101-128.

Mazza, J. C. (2003). Mediciones antropométricas. Estandarización de las técnicas de medición, actualizada según parámetros internacionales. PubliCE Standard.

Norton, K. \& Olds, T. (1996). Antropométrica. UNSW Press.

Pai, C., Hay J., \& Wilson, B. (1984). Stroking Techniques of Elite Swimmers. Journal of Sports Sciences, (2), 225-239.

Rienzi, E., Mazza J., Carter, J. \& Reilly T. (Eds) (1995). Futbolista sudamericano de elite: morfología, análisis del juego y performance. Biosystem Servicio Educativo.

Rivera, M. \& Avella, F. (1992). Características antropométricas y fisiológicas de futbolistas puertorriqueños. Archivos de Medicina del Deporte, IX (35), 265-277.

Ross, W., De Rose, E. \& Ward, R. (1988). Anthropometry Applied to Sport Medicine. In: edited by A. Dirix, H.G. Knuttgen and T.K. Tittel. The Olympic Book Of Sports Medicine. Blackwell Scientific Publications, pp. 233-265.

Thayer, A. L., \& Hay, J. G. (1984). Motivating start and turn improvement. Swimming Technique, 20(4), 17-20.

Wilke K. \& Madsen O. (1990). El Entrenamiento del Nadador Juvenil. 1 ed. Stadium. 